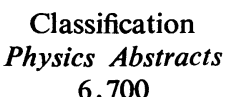

\title{
ÉTUDE CINÉTIQUE ET ÉNERGÉTIQUE DE LA DISSOCIATION DE L'HÉMIOXYDE D'AZOTE DANS UN CHAMP ÉLECTRIQUE ALTERNATIF
}

\author{
R. BES \\ Laboratoire de Chimie-Physique et Electrochimie \\ Institut du Génie Chimique 31078 Toulouse Cedex, France
}

(Reçu le 8 novembre 1976, révisé le 24 février 1977, accepté le 22 mars 1977)

\begin{abstract}
Résumé. - On étudie la dissociation de l'hémioxyde d'azote dans un champ électrique alternatif non-uniforme pour des valeurs du champ réduit comprises entre 3 et $60 \times 10^{-16}$ volt. $\mathrm{cm}^{2}$. molécule $^{-1}$. L'analyse des résultats expérimentaux dans le cadre de la théorie cinétique des réactions chimiques induites dans les décharges électriques dans les gaz, fait apparaître effectivement une énergie minimale de dissociation électrique, trouvée ici égale à $2,7 \pm 0,6 \mathrm{eV}$.molécule ${ }^{-1}$, pour une valeur particulière du champ électrique réduit, dans le cas présent comprise entre 7 et $9 \times 10^{-16}$ volt. $\mathrm{cm}^{2}$. molécule ${ }^{-1}$. Les données cinétiques et énergétiques s'accordent avec un mécanisme de dissociation dans lequel le premier état triplet répulsif $\left(\begin{array}{l}3 \\ 1\end{array}\right)$ joue le rôle de complexe activé :
\end{abstract}

$$
\mathrm{N}_{2} \mathrm{O}\left({ }^{1} \Sigma^{+}\right) \frac{\text { impact }}{\text { electronique }} \mathrm{N}_{2} \mathrm{O}\left(\begin{array}{l}
3 \\
1
\end{array}\right) \longrightarrow \mathrm{N}_{2}\left({ }^{1} \Sigma_{\mathrm{g}}^{+}\right)+0\left({ }^{3} \mathrm{P}_{\mathrm{g}}\right)
$$

\begin{abstract}
The electrical breakdown of nitrous oxide in an alternating nonuniform field has been studied in the range 3-60 $\times 10^{-16}$ volt. $\mathrm{cm}^{2}$. molecule ${ }^{-1}$ for the reduced field. The experimental results have been treated according to the kinetic theory of chemical reactions in gaseous electrital discharges. The predicted minimum for the electrical dissociation energy, here equal to $2.7 \pm 0.6 \mathrm{eV}$. molecule -1 , has been found in the range 7-9 $\times 10^{-16}$ volt. $\mathrm{cm}^{2}$. molecule ${ }^{-1}$ for the reduced electrical field. The kinetic and energetic data agree with a dissociation mechanism in which the first repulsive triplet state $\left({ }_{1}^{3} \Pi\right)$ acts as the activated complex :
\end{abstract}

$$
\mathrm{N}_{2} \mathrm{O}\left({ }^{1} \Sigma^{+}\right) \stackrel{\text { electronic }}{\text { impact }} \mathrm{N}_{2} \mathrm{O}\left({ }_{1}^{3} \Pi\right) \longrightarrow \mathrm{N}_{2}\left({ }^{1} \Sigma_{\mathrm{g}}^{+}\right)+0\left({ }^{3} \mathrm{P}_{\mathrm{g}}\right) \text {. }
$$

1. Introduction. - La décharge électrique dans les gaz est souvent utilisée pour produire des réactions chimiques difficiles à réaliser à la température ambiante. Parmi les plus importantes sur le plan industriel, citons les synthèses d'ozone, d'acétylène, de monoxyde d'azote et d'hydrazine, ainsi que les polymérisations de molécules organiques. Des réactions de décomposition peuvent également être induites dans un réacteur à plasma. Dans ce laboratoire, la conversion des oxydes d'azote $\mathrm{NO}$ [1], $\mathrm{NO}_{2}$ [2] et $\mathrm{N}_{2} \mathrm{O}$ [3] en azote et oxygène moléculaires a été effectuée. Des études cinétiques portant sur ces réactions ont été réalisées dans un large domaine de variation du paramètre $N_{0} . d$ (concentration initiale $X$ distance interélectrodes); elles ont permis de montrer le rôle fondamental de la tension et de la fréquence imposées dans le cas d'une décharge électrique en champ alternatif et de dégager l'expression de la constante de vitesse apparente de dissociation [4].

La théorie cinétique des réactions chimiques induites dans les décharges électriques [5] prévoit un maximum d'efficacité dans l'utilisation de l'énergie apportée par le champ électrique $E$. En d'autres termes, l'énergie effectivement consommée dans la réaction chimique observée est minimale pour une certaine valeur du champ électrique réduit $E / N_{0}$, paramètre fondamental qui détermine l'énergie moyenne des électrons du plasma. Ce phénomène a été mis en évidence expérimentalement au Laboratoire dans le cas de la dissociation primaire du chlorure de nitrosyle $\mathrm{NOCl}$ [6]. Le mécanisme cinétique de dissociation de cette entité chimique n'a pu être complètement déterminé par suite de l'inexistence dans les tables de sections efficaces électroniques et coefficients macroscopiques [7], des données relatives à cette molécule. En ce qui concerne les trois oxydes d'azote $\mathrm{NO}, \mathrm{NO}_{2}$ et $\mathrm{N}_{2} \mathrm{O}$ et, bien que quelques données existent déjà [7], l'abondance subite des publications spécifiques au cours de ces cinq dernières années, amène à penser que le mécanisme de dissociation de ces espèces sous l'impact électronique pourra être enfin élucidé.

Nous présentons dans ce travail une étude systématique de l'influence du champ électrique réduit sur l'énergie effectivement consommée lors de la dissociation primaire de l'hémioxyde d'azote $\mathrm{N}_{2} \mathrm{O}$ dans une décharge alternative afin de trouver, s'il existe, le minimum énergétique prévu par la théorie. A ce titre, 
ce texte développe et complète une première note [8] : le paramètre expérimental $N_{0} . d$ varie ici entre $6 \times 10^{17}$ et $2 \times 10^{19}$ molécule. $\mathrm{cm}^{-2}$. Nous discutons ensuite d'un mécanisme réactionnel compatible aux données cinétiques et énergétiques.

2. Appareillage et techniques expérimentales. L'appareillage est dans son ensemble identique à celui précédemment décrit [1]; il comporte trois parties essentielles : le dispositif d'alimentation électrique, la cellule de décomposition ou effluveur, et le système de mesure et d'enregistrement de la pression régnant dans l'enceinte effluvée.

On peut faire varier les paramètres électriques dans

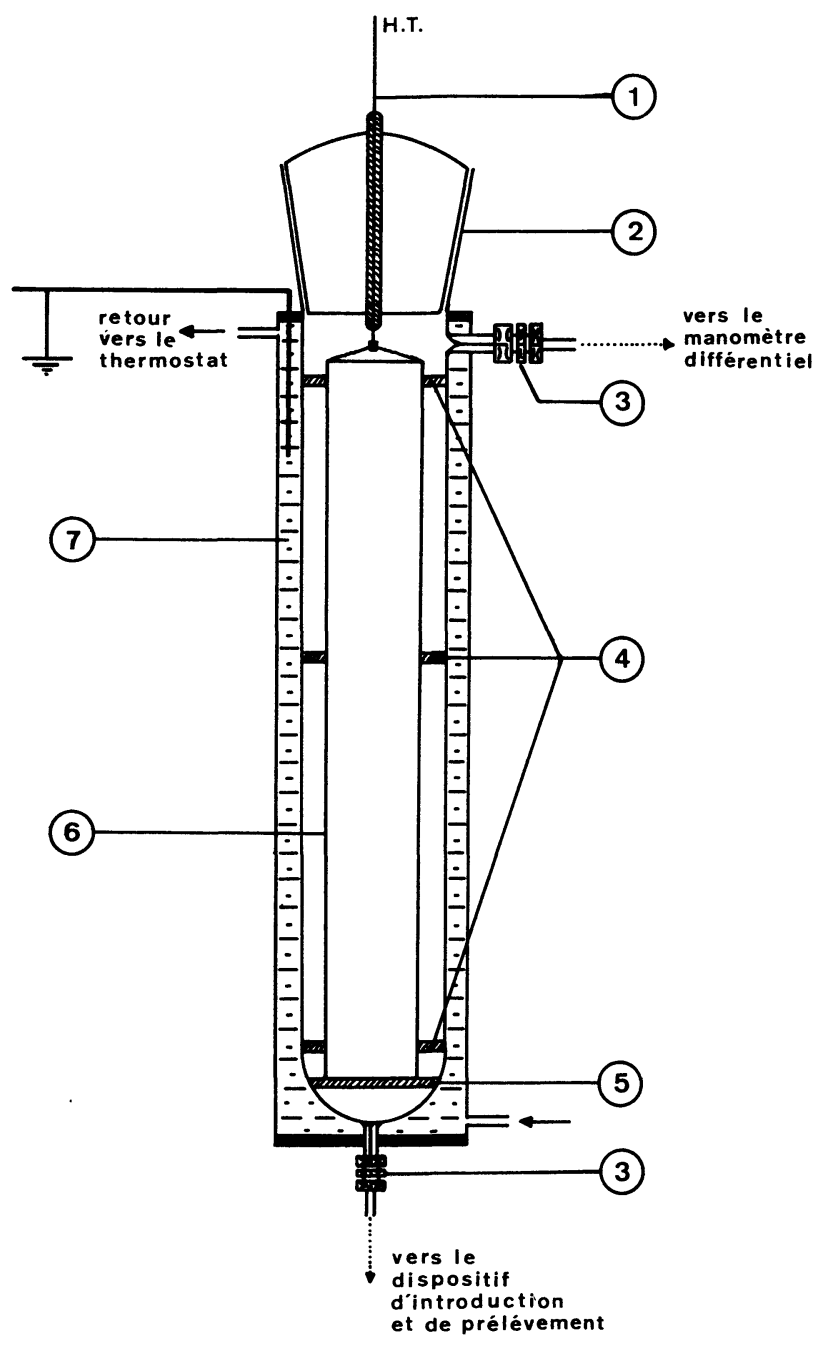

Fig. 1. - Effluveur comportant une électrode métallique : (1) Alimentation haute tension; (2) Rodage 34-36 à passage fermé (le fil d'amenée de courant qui le traverse est en tungstène) ; (3) Raccord Swagelok en acier inoxydable ; (4) Bague de centrage en Téflon ; (5) Grille support en Téflon ; (6) Cylindre métallique, plein ou grillagé, constituant l'électrode haute-tension ; (7) solution saline constituant l'électrode portée à la terre.

[Discharge reactor with a metallic electrode : 1) high voltage. 2) 34/36 standard taper joint (the crossing wire is of tungsten). 3) Stainless steel Swagelok junction. 4) Teflon spacer. 5) Teflon gate. 6) Metallic high voltage electrode : cylindrical grid or whole cylinder. 7) grounded electrode : sodium chloride solution.] les gammes suivantes : $0-22,5 \mathrm{kV}$, pour la tension efficace $V ; 50-1500 \mathrm{~Hz}$ pour la fréquence $F$ du courant. On a utilisé ici deux types d'effluveurs : dans le premier, le gaz soumis à l'effluve est contenu dans l'espace annulaire compris entre deux cylindres de verre Pyrex coaxiaux constituant les armatures d'électrodes renfermant une solution aqueuse de chlorure de sodium [1]; dans le second type d'effluveur, on a remplacé le tube interne de verre par une électrode métallique cylindrique, pleine ou grillagée. La figure 1 donne une illustration de ce dernier type d'effluveur. Pour tous les effluveurs, la circulation forcée de la solution saline constituant l'électrode externe, portée à la terre, et l'utilisation conjuguée d'un thermostat assurent le maintien de l'ensemble à une température fixe, choisie dans le domaine $\left[-6,+60^{\circ} \mathrm{C}\right]$.

Les caractéristiques géométriques et électriques des effluveurs utilisés sont rassemblées dans le tableau I. A cet égard, rappelons qu'il est admis [9] que l'effluveur se comporte comme une suite de condensateurs en série; nous désignerons par $C_{\mathrm{G}}$ la capacité de l'espace interélectrodes, $C_{\mathrm{D}}$ la capacité du diélectrique de verre et $C_{\mathrm{T}}$ la capacité totale du réacteur. Celle-ci est obtenue à partir des caractéristiques tensioncourant déterminées avant le régime de décharge. La capacité $C_{\mathrm{D}}$ est mesurée de manière similaire en courtcircuitant la capacité $C_{\mathrm{G}}$.

L'hémioxyde d'azote utilisé provient d'une bouteille du commerce; la composition volumique du gaz indiquée par le fabricant est la suivante :

$\begin{array}{ll}\mathrm{N}_{2} \mathrm{O}>99,5 \% ; & \mathrm{O}_{2}<50 \text { p. p. m. } \\ \mathrm{H}_{2} \mathrm{O}<20 & \text { p. p. m. } ; \quad \mathrm{N}_{2}<5000 \text { p. p. m. } \\ \mathrm{H}_{2}<1 & \text { p. p. m. } ; \mathrm{CO}<2 \text { p. p. m. } \\ \text { et } \quad & \\ \mathrm{CO}_{2}<2 & \text { p. p. m. }\end{array}$

Les analyses chimiques des échantillons prélevés sont effectuées à l'aide d'un spectromètre de masses. En ce qui concerne l'hémioxyde d'azote pur, le spectre obtenu est en parfait accord avec celui indiqué dans les tables [10] et le degré réel de pureté est de $(99,7 \pm 0,1) \%$ en volume.

Une fois introduit l'hémioxyde d'azote dans l'effluveur, la décomposition a lieu en système fermé. L'enregistrement continu de la pression $P$ au cours de l'expérience fournit les valeurs de la vitesse initiale de décomposition $v_{0}$ à partir des pentes $\mathrm{d} P / \mathrm{d} t$ mesurées à l'instant initial. La dissociation s'accompagne en effet d'une variation de pression compte tenu de la réaction globale [3] :

$$
\mathrm{N}_{2} \mathrm{O} \rightarrow \mathrm{N}_{2}+\frac{1}{2} \mathrm{O}_{2} .
$$

En fait, on observe dès la mise sous tension de l'effluveur, une brutale augmentation de la pression qui n'a aucun rapport avec la dissociation proprement dite. En effet, si l'on porte les valeurs de la variation de la pression $\Delta P$ en fonction du temps durant les dix secondes suivant la mise sous tension de l'effluveur, 


\section{TABLEAU I}

Caractéristiques géométriques et électriques des effluveurs Epaisseur moyenne du verre : 1,6 $\pm 0,1 \mathrm{~mm}$, Hauteur : $\sim 65 \mathrm{~cm}$

\begin{tabular}{lcc}
\multicolumn{1}{c}{ Effluveur } & $\begin{array}{c}\text { Rayon interne } \\
\text { de l'électrode } \\
\text { externe }(\mathrm{mm})\end{array}$ & $\begin{array}{c}\text { Volume gazeux } \\
\text { total }: \tau\left(\mathrm{cm}^{3}\right)\end{array}$ \\
$\begin{array}{l}\text { Type I } \\
\text { Verre-verre }\end{array}$ & 15,5 & $-54,5$ \\
& 17,4 & 146 \\
& 12,8 & 151 \\
& 12,1 & 193 \\
Type II & 12,8 & 185 \\
Verre-laiton & 12,8 & 308 \\
& 13,1 & 253 \\
Verre-Aluminium & 5,60 & 56 \\
& 5,60 & 60 \\
& 5,60 & 66 \\
Verre-tungstène & 20,9 & 736 \\
& 5,60 & 66 \\
Verre-acier inoxyda- & 20,9 & 431 \\
ble & 20,9 & 585 \\
& 20,9 & 682 \\
& 20,9 & 736 \\
& 3,15 & 18 \\
& 5,60 & 66 \\
& 10,4 & 198 \\
& 5,60 & 66 \\
& 15,5 & 396 \\
& 15,5 & 396 \\
& 15,5 & 295
\end{tabular}

on obtient une variation linéaire dont l'extrapolation au temps origine conduit à une valeur finie positive. Un phénomène analogue a d'ailleurs été observé dans le cas de la dissociation primaire du dioxyde de carbone $\mathrm{CO}_{2}$ dans une décharge électrique luminescente [11]. L'augmentation quasi instantanée de la pression à la mise sous tension de l'effluveur a alors été interprétée comme étant due à la désorption de quelques molécules de $\mathrm{CO}_{2}$ adsorbées sur les parois, sous l'effet du bombardement électronique. Dans notre cas, on constate à l'arrêt de l'effluve le phénomène inverse, c'est-à-dire une brutale diminution de la pression ; de plus, l'amplitude des deux variations est beaucoup plus importante sur les effluveurs du type II.

Les analyses chimiques indiquent [3] que l'hémioxyde d'azote est dissocié sous l'effet du bombardement électronique suivant les deux réactions globales parallèles du premier ordre :

$$
\begin{aligned}
& \mathrm{N}_{2} \mathrm{O} \stackrel{k_{1}}{\longrightarrow} \mathrm{N}_{2}+\frac{1}{2} \mathrm{O}_{2} \\
& \mathrm{~N}_{2} \mathrm{O} \stackrel{k_{2}}{\longrightarrow} \mathrm{NO}+\frac{1}{2} \mathrm{~N}_{2}
\end{aligned}
$$

et nous avons trouvé par spectrométrie de masse $k_{1}=2 k_{2}[3]$.

Le monoxyde d'azote NO est ensuite dissocié en azote et oxygène selon un processus identique à celui trouvé dans le cas où ce composé est pur [1].

\begin{tabular}{cccc}
$\begin{array}{c}\text { Distance } \\
\text { interélectrodes } \\
d=\tau / \mathrm{S}(\mathrm{mm})\end{array}$ & & & \\
$S \begin{array}{c}\text { surface moyenne } \\
\text { des électrodes }\end{array}$ & $C_{\mathrm{T}}$ & $\begin{array}{c}C_{\mathrm{D}} \\
\left(10^{-12} \mathrm{~F}\right)\end{array}$ & $\begin{array}{c}\left(10^{-12} \mathbf{F}\right) \\
C_{\mathbf{D}} / \mathbf{S} \\
\left(10^{-12} \cdot \mathbf{c m}^{-2}\right)\end{array}$ \\
\hline 0,6 & - & - & - \\
2,4 & 430 & 811 & 1,41 \\
2,9 & 151 & 625 & 1,02 \\
3,2 & 111 & 599 & 1,15 \\
3,8 & 95 & 688 & 1,14 \\
7,0 & 82 & 550 & 1,13 \\
7,2 & 43 & 418 & 0,95 \\
2,6 & 43 & 404 & 1,15 \\
3,6 & 63 & 749 & 4,74 \\
4,5 & 36 & 749 & 5,24 \\
15,0 & 27 & 749 & 5,76 \\
4,5 & 31 & 2183 & 4,97 \\
5,9 & 27 & 749 & 5,76 \\
10,0 & 103 & 2183 & 3,83 \\
13,0 & 59 & 2183 & 4,28 \\
15,0 & 35 & 2183 & 4,76 \\
2,1 & 31 & 2183 & 5,06 \\
4,5 & 42 & 468 & 5,92 \\
9,3 & 27 & 749 & 5,76 \\
& 20 & 1113 & 4,78 \\
4,5 & & & \\
4,5 & 27 & 749 & 5,76 \\
4,9 & 120 & 1740 & 2,17 \\
7,5 & 175 & 1410 & 0,98 \\
& 51 & 1536 & 3.92 \\
& & &
\end{tabular}

Selon les conditions électriques, le dioxyde d'azote $\mathrm{NO}_{2}$ peut apparaître au sein du mélange gazeux par suite de la réaction thermochimique d'oxydation du monoxyde d'azote par l'oxygène [3]. Notons que la séquence des réactions (2) et (3) a été confirmée dernièrement dans une décharge luminescente et en régime dynamique [12].

La constante de vitesse apparente de dissociation $k$ $\left(\mathrm{s}^{-1}\right)$, égale à la somme des constantes de vitesse apparentes $k_{1}$ et $k_{2}$ des réactions (2) et (3), est calculée par la relation :

$$
k=\frac{v_{0}}{N_{0}}
$$

où $N_{0}$ désigne la concentration initiale de l'hémioxyde d'azote (molécule. $\mathrm{cm}^{-3}$ ) et $v_{0}$ est la vitesse initiale de dissociation

$$
\left(v_{0}=-\frac{\mathrm{d}\left[\mathrm{N}_{2} \mathrm{O}\right]}{\mathrm{d} t}\right) .
$$

En négligeant la diffusion des porteurs de charge et en admettant l'hypothèse suivant laquelle la mobilité des ions positifs ou négatifs est négligeable devant celle des électrons [13], l'expression de la constante de vitesse apparente de dissociation est [4] :

$$
k=4 \sqrt{2} \cdot \frac{\alpha_{\mathrm{d}}}{q \cdot W} \cdot \frac{C_{\mathrm{D}}}{S}\left(V-\frac{C_{\mathrm{G}}}{C_{\mathrm{T}}} V_{0}\right) F
$$


avec :

$\alpha_{d}$, constante de vitesse réelle de dissociation (molécule ${ }^{-1} \cdot \mathrm{cm}^{3} \cdot \mathrm{s}^{-1}$ );

$q$, charge de l'électron;

$W$, vitesse induite moyenne des électrons dans le sens du champ électrique ;

$S$, surface moyenne des électrodes ;

$V$ et $V_{0}$, valeurs efficaces respectives de la tension appliquée et du potentiel de maintien de la décharge électrique dans le gaz; $V_{\mathrm{S}}=C_{\mathrm{G}} / C_{\mathrm{T}} \cdot V_{0}$ est le potentiel de seuil ;

$F$, fréquence fondamentale du courant.

Le potentiel de maintien $V_{0}$ est déterminé par la méthode du parallélogramme [14], mise en œuvre ici sur chaque effluveur et pour toute valeur de $N_{0}$ (Fig. 2).

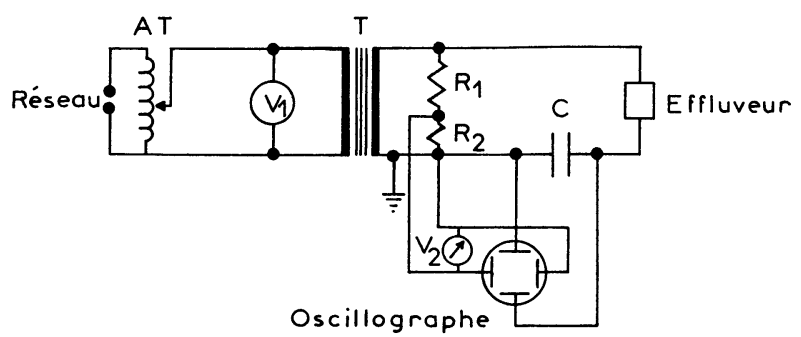

Fig. 2. - Alimentation électrique (pour $F \neq 50 \mathrm{~Hz}$, voir la référence [7]) et dispositif de mesure de la puissance dissipée dans le gaz. Pont de mesure de la tension secondaire: $R_{1}=60 \mathrm{M} \Omega, R_{2}=60 \mathrm{k} \Omega$ (résistances non inductives); C, condensateur de mesure de la quantité d'électricité : $1 \mu \mathrm{F} . \mathrm{V}_{1}$ et $V_{2}$, voltmètres électroniques. Le potentiel de maintien $V_{0}$ représente la demi-largeur du parallélogramme suivant l'axe des tensions.

[Electrical supply (for $F \neq 50 \mathrm{~Hz}$, see Ref. [7]) and power consumption device. Resistance bridge for the measurement of the secondary voltage : $R_{1}=60 \mathrm{M} \Omega, R_{2}=60 \mathrm{k} \Omega$. Capacitor for the measurement of the electrical charge : $C=1 \mu \mathrm{F}$. $\mathrm{V}_{1}$ and $V_{2}$, electronic voltmeters. The sustaining voltage is the half-width of the parallelogram along the voltage axis.]

L'utilisation des relations (4) et (5) conduit ainsi à la valeur du rapport $\alpha_{\mathrm{d}} / W$ et de là, à l'énergie de dissociation $\left(\mathrm{eV}\right.$. molécule $\left.^{-1}\right)$ par la relation $[5,8]$ :

$$
\varepsilon=\frac{\sqrt{2} \cdot q \cdot W}{\alpha_{\mathrm{d}}} \cdot \frac{E}{N_{0}}
$$

dans laquelle on a posé le champ électrique $E$ égal à $V_{0} / d$ ( $d$ est la distance interélectrodes), c'est-à-dire que l'on considère ici la valeur moyenne du champ, comme celá est d'usage courant dans de telles études [15].

3. Résultats et discussion. - La figure 3 indique la variation de $\varepsilon_{\mathrm{N}_{2} \mathrm{O}}$ en fonction du champ électrique réduit $\left(E / N_{0}\right)$. Avant de poursuivre, il est important de noter que l'erreur relative théorique sur la valeur de $\varepsilon_{\mathrm{N}_{2} \mathrm{O}}$ calculée selon la séquence des relations (4), (5) et (6) est voisine de $30 \%$; en fait, la dispersion des valeurs expérimentales de $\varepsilon_{\mathrm{N}_{2} \mathrm{O}}$, pour le même champ réduit et sur le même effluveur, ne dépasse jamais

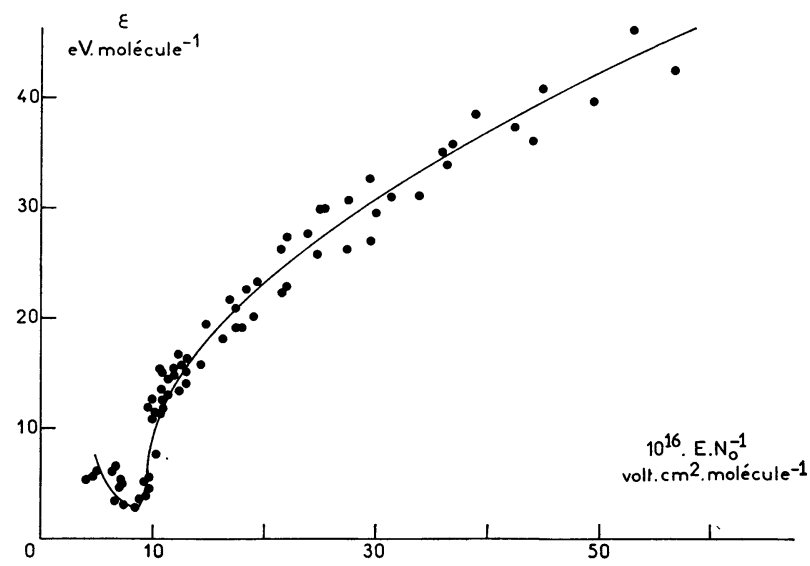

FIG. 3. - Variation de l'énergie de dissociation électrique du $\mathrm{N}_{2} \mathrm{O}$ en fonction du champ électrique réduit.

[Variation of electrical dissociation energy of $\mathrm{N}_{2} \mathrm{O}$ with reduced field.]

$20 \%$ de la valeur moyenne. Par ailleurs, à champ réduit donné, $\varepsilon_{\mathrm{N}_{2} \mathrm{O}}$ conserve la même valeur lorsque l'on passe d'un effluveur du type I à un effluveur du type II. Dans ces conditions, $\varepsilon_{\mathrm{N}_{2} \mathrm{O}}$ apparaît comme une fonction univoque du champ électrique réduit, dans le domaine envisagé, et ce, conformément à la théorie cinétique de Lunt [5]. On constate de plus que $\varepsilon_{\mathrm{N}_{2} \mathrm{O}}$ passe par une valeur minimale et égale à

$$
2,7 \pm 0,6 \mathrm{eV} \cdot \text { molécule }^{-1}
$$

pour un champ réduit compris entre 7 et

$$
9 \times 10^{-16} \text { volt } . \mathrm{cm}^{2} \cdot \text { molécule }{ }^{-1} \text {. }
$$

On obtient ainsi une nouvelle confirmation expérimentale de l'existence d'un maximum d'efficacité dans l'utilisation de l'énergie fournie par le champ électrique par le biais des électrons ; en d'autres termes et en accord avec Lunt [5], la valeur minimale de $\varepsilon$ caractérise l'aspect économique de la décharge électrique en tant que procédé pour réaliser une réaction chimique.

A notre connaissance, les seules autres réactions étudiées à ce jour et présentant le même phénomène sont la dissociation de l'hydrogène [16], la synthèse de l'eau [17] ainsi que la dissociation du chlorure de nitrosyle [6]. Un mécanisme de réaction a été proposé dans le seul cas de l'hydrogène [18] où la dissociation résulte du passage de la molécule de son état fondamental au premier état triplet répulsif ${ }^{3} \Sigma_{\mathbf{u}}^{+}$. La section efficace correspondante, nulle en deçà de $8,8 \pm 0,2 \mathrm{eV}$, présente un maximum très prononcé pour une énergie des électrons égale à $16,5 \mathrm{eV}$. Remarquons que les travaux cités en référence [16], exploités dans ce sens, permettent de situer la valeur minimale de $\varepsilon_{\mathrm{H}_{2}}$ entre 15,4 et $17,2 \mathrm{eV}$. Il y a donc un parfait accord entre ces diverses déterminations de la valeur la plus économique de l'énergie de dissociation électrique de la molécule d'hydrogène en ses atomes.

Pour $\mathrm{N}_{2} \mathrm{O}$, on se propose de tester les différents 
intermédiaires des réactions $(2-3)$ qu'il est possible d'imaginer, c'est-à-dire soit un ion négatif temporaire $\left(\mathrm{N}_{2} \mathrm{O}\right)^{-}$, soit un état répulsif $\left(\mathrm{N}_{2} \mathrm{O}\right)^{*}$, soit enfin un ion positif $\left(\mathrm{N}_{2} \mathrm{O}\right)$.

Le calcul de l'énergie moyenne des électrons dans la décharge peut être effectué de deux manières. La première, introduite par l'école de Townsend [19], fait appel aux mesures de l'énergie caractéristique et de la vitesse induite moyenne des électrons dans le gaz considéré. Dans le cas du $\mathrm{N}_{2} \mathrm{O}$, ces mesures [20] couvrent le domaine de champ réduit allant environ de 0,3 à $10 \times 10^{-16}$ volt. $\mathrm{cm}^{2}$. molécule ${ }^{-1}$, c'est-àdire pratiquement toute la partie gauche de la courbe de la figure 3 . Entre 4 et $10 \times 10^{-16}$ volt. $\mathrm{cm}^{2}$. molécule $^{-1}$, on trouve que l'énergie moyenne des électrons $\bar{\varepsilon}_{\mathrm{e}}$ varie entre 1 et $3 \mathrm{eV}$. La seconde méthode d'estimation de $\bar{\varepsilon}_{\mathrm{e}}$ fait appel à la relation de Von Engel [21] :

$$
\frac{\exp x}{x^{1 / 2}}=1,2 \times 10^{7}(\mathrm{cpR})^{2}
$$

avec $x=q \cdot V_{\mathrm{i}} / k T_{\mathrm{e}} ; q$ est la charge de l'électron, $V_{\mathrm{i}}$ le potentiel d'ionisation du gaz (volts), $k$ est la constante de Boltzmann, $T_{\mathrm{e}}$ la température électronique moyenne $(\mathrm{K})$, c est une constante dépendant de la nature du gaz $\left(10^{-2}-5 \times 10^{-2}\right)$, p, la pression du gaz (torrs) et $R$, la distance interélectrodes $(\mathrm{cm})$. Dans nos conditions de travail où la pression varie entre 6 et 600 torrs, on trouve que $T_{\mathrm{e}}$ est comprise entre 7500 et $15500 \mathrm{~K}$. L'énergie moyenne des électrons se déduit immédiatement de ces valeurs par la relation $q V_{\mathrm{i}}=k T_{\mathrm{e}}$ : on trouve ainsi que $\bar{\varepsilon}_{\mathrm{e}}$ est comprise entre 1 et $2 \mathrm{eV}$.

En résumé, les deux méthodes d'estimation de l'énergie moyenne des électrons sont en accord et nous retiendrons par la suite la valeur $\bar{\varepsilon}_{\mathrm{e}}=2 \mathrm{eV}$. Remarquons que c'est là une valeur bien faible par rapport à l'énergie de première ionisation du $\mathrm{N}_{2} \mathrm{O}: 12,9 \mathrm{eV}$ [7]. Afin de mieux comprendre le rôle des électrons lors des chocs électron-molécule, nous avons calculé les courbes de répartition énergétique à partir des distributions de Maxwell et de Druyvesteyn [21]. On sait en effet que la distribution réelle des électrons dans la décharge électrique est comprise entre ces deux distributions limites [22]; on s'aperçoit ici qu'environ $0,2 \%$ des électrons sont capables d'ioniser les molécules selon un processus d'extraction d'un électron. De plus, on sait qu'à une énergie des électrons de $14 \mathrm{eV}$, la section efficace pour l'ionisation est seulement de $0,54 \times 10^{-17} \mathrm{~cm}^{2}$ alors que la section efficace totale est d'environ $1,5 \times 10^{-15} \mathrm{~cm}^{2}$ [7]. Au total, l'intermédiaire $\left(\mathrm{N}_{2} \mathrm{O}\right)^{+}$ne joue aucun rôle déterminant dans le mécanisme intime de dissociation de la molécule de $\mathrm{N}_{2} \mathrm{O}$.

Il nous reste maintenant à envisager le cas de $\left(\mathrm{N}_{2} \mathrm{O}\right)^{-}$ et $\left(\mathrm{N}_{2} \mathrm{O}\right)^{*}$. L'attachement électronique s'effectue généralement à basse énergie $(\leqslant 5 \mathrm{eV})$, c'est-à-dire dans un domaine où la probabilité d'existence des électrons dans la décharge est très grande. Dans le cas de l'hémioxyde d'azote, l'ion $\left(\mathrm{N}_{2} \mathrm{O}\right)^{-}$n'a jamais été for- mellement identifié et l'on observe effectivement un attachement dit dissociatif par suite de la formation de l'ion $\left(\mathrm{O}^{-}\right)$:

$$
\mathrm{e}+\mathrm{N}_{2} \mathrm{O} \stackrel{k^{\prime}}{\rightarrow} \mathrm{N}_{2}+\mathrm{O}^{-} .
$$

Les études cinétiques [23] effectuées par plusieurs équipes s'accordent à la valeur $k^{\prime}=4 \times 10^{-15}$ molécule ${ }^{-1} \cdot \mathrm{cm}^{3} \cdot \mathrm{s}^{-1}$. A titre de comparaison, les valeurs de la vitesse induite moyenne des électrons dont nous disposons dans le domaine de champ électrique réduit allant de 4 à $10 \times 10^{-16}$ volt. $\mathrm{cm}^{2}$. molécule ${ }^{-1}$ [7] permettent de calculer la valeur moyenne de la constante de vitesse réelle de dissociation $\alpha_{d}$ (cf. relations 4 et 5): on trouve $3,5 \times 10^{-9}$ molécule ${ }^{-1} \cdot \mathrm{cm}^{3} \cdot \mathrm{s}^{-1}$. En conséquence, il est fortement improbable que le processus (8) intervienne dans la décharge et par là même que l'ion temporaire $\left(\mathrm{N}_{2} \mathrm{O}\right)^{-}$joue un rôle dans la réaction de dissociation de l'hémioxyde d'azote.

$\mathrm{Au}$ total, il ne demeure qu'une seule possibilité quant à la nature du complexe activé, en l'occurrence un état excité répulsif $\left(\mathrm{N}_{2} \mathrm{O}\right)^{*}$, et il s'agit de savoir si cette hypothèse est compatible aux données cinétiques et énergétiques dont nous disposons. Dès l'abord, il importe de remarquer que l'énergie minimale de dissociation de l'hémioxyde d'azote mise en exergue dans ce travail $(2,7 \pm 0,6 \mathrm{eV})$ correspond pratiquement à l'énergie d'activation mesurée lors de la dissociation thermique : $60 \mathrm{kcal} / \mathrm{mole}$ soit environ $2,6 \mathrm{eV}$ [24]. Dans ce cas, la dissociation unimoléculaire a été interprétée comme étant due au processus

$$
\mathrm{N}_{2} \mathrm{O}\left({ }^{1} \Sigma^{+}\right) \rightarrow \mathrm{N}_{2} \mathrm{O}\left({ }^{3} \Pi\right) \rightarrow \mathrm{N}_{2}\left({ }^{1} \Sigma_{\mathrm{g}}^{+}\right)+\mathrm{O}\left({ }^{3} \mathrm{P}_{\mathrm{g}}\right)
$$

c'est-à-dire le passage de la molécule $\mathrm{N}_{2} \mathrm{O}$ de son état fondamental singulet à l'état triplet répulsif ${ }^{3} \Pi$ le long de l'axe de la liaison NN-O. La réaction (9) est certainement prépondérante dans la décharge électrique car dans l'hypothèse d'un processus primaire d'activation électronique, il est normal de trouver - et nous en avons eu un exemple dans le cas de l'hydrogène - une énergie minimale de dissociation égale à celle assurant le passage de la molécule de son état fondamental au premier (le plus bas) état excité.

Remarquons enfin que le comportement identique des effluveurs du point de vue énergétique amène à retenir pour la mise en œuvre de la réaction de dissociation en régime dynamique, un effluveur du type II. En effet, l'observation de la relation (5) montre que pour une valeur donnée de $E / N_{0}-\alpha_{\mathrm{d}} / W$ est alors connu à fréquence $\mathrm{F}$ fixe et pour un même écart de tension $\Delta V=V-V_{\mathrm{s}}$, l'effluveur du type II conduit à une meilleure cinétique que l'effluveur du type I de même encombrement par suite de l'augmentation importante du rapport $C_{\mathrm{D}} / S$. En ce qui concerne la nature du métal constituant l'électrode interne des effluveurs du type II, le critère de choix est fourni à partir du tracé des courbes de Paschen : $V_{0}=f\left(N_{0} . d\right)$. Pour ce faire, on construit les variations $E / N_{0}=f\left(N_{0} . d\right)$; l'intersection de ces courbes avec l'horizontale $\left(E / N_{0}\right)_{\min }$ correspon- 
dant à l'énergie minimale de dissociation électrique permet donc de déterminer le potentiel de maintien caractéristique de chaque électrode: on retient le métal imposant la plus faible valeur de $V_{0}$. Dans le cas présent, il n'y a pas de différence majeure entre les matériaux utilisés ; cela peut s'expliquer dans la mesure où ceux-ci présentent des potentiels d'extraction des électrons à peu près identiques $(4-5 \mathrm{eV})$.

4. Conclusion. - L'étude cinétique et énergétique de la dissociation primaire de l'hémioxyde d'azote dans un champ électrique alternatif amène une nouvelle confirmation expérimentale de la théorie cinétique de Lunt. L'énergie de dissociation électrique est bien une fonction univoque du champ électrique réduit et présente une valeur minimale. Cette valeur, $2,7 \pm 0,6 \mathrm{eV}$. molécule ${ }^{-1}$, s'accorde parfaitement avec l'énergie d'activation mesurée lors de la dissociation thermique, soit $2,6 \mathrm{eV}$. molécule ${ }^{-1}$. En conséquence, le mécanisme le plus probable de dissociation de l'hémioxyde d'azote dans un champ électrique alternatif est identique.

\section{Bibliographie}

[1] Bes, R., Lacoste, G. et Mahenc, J., J. Chim. Phys. 67 (1970) 731.

[2] Lacoste, G., Bes, R. et Mahenc, J., J. Chim. Phys. 67 (1970) 736.

[3] Bes, R., Lacoste, G. et El Khemiri, H., J. Chim. Phys. 70 (1973) 433.

[4] Lacoste, G. et Bes, R., Rev. Chim. Min. 11 (1974) 141.

[5] Lunt, R., Adv. Chem. Ser. 80 (1969) 452.

[6] Savall, A., Lacoste, G. et Routie, R., C. R. Hebd. Séan. Acad. Sci. C 277 (1973) 603.

[7] Laborie, P., Rocard, J. M. et Rees, J. A., Sections efficaces électroniques et coefficients macroscopiques; vol. II : Vapeurs métalliques et gaz moléculaires; (Dunod, Paris), 1971.

[8] Bes, R. et Routie, R., C. R. Hebd. Séan. Acad. Sci. C 282 (1976) 869.

[9] Katakis, D. et Bersis, D., J. Appl. Phys. 36 (1965) 1298.

[10] Cornu, A. et Massot, R., Index de Spectres de Masse : (Heyden and son et P. U. F., London-Paris), 1966; Begun, G. M. et Landau, L., J. Chem. Phys. 35 (1961) 547.

[11] Corvin, K. K. et Corrigan, S. J. B., J. Chem. Phys. 50 (1969) 2570.

[12] Austin, J. M. et Smith, A. L. S., J. Phys. D 6 (1973) 2236.

[13] Kaufman, F., Adv. Chem. Ser. 80 (1969) 29.
[14] Manley, T. C., Trans. Electrochem. Soc. 84 (1943) 83.

[15] ReIN, A., Electra 32 (1974) 43.

[16] Poole, H. G., Proc. Roy. Soc. A 163 (1937) 404, 415 et 424 ; Shaw, T. M., J. Chem. Phys. 30 (1959) 1366.

[17] Kirkby, P. J., Phil. Mag. 13 (1907) 289 ; Proc. Roy. Soc. A 85 (1910) 151.

[18] Corrigan, S. J. B., J. Chem. Phys. 43 (1965) 4381.

[19] Loeb, L. B., Fundamental Processes of Electrical Discharges in gases ; (Wiley, New-York) 1939.

[20] Cf. McDaniel, E. W., Collision Phenoma in Ionized gases (Wiley, New-York) 1964, p. 522-546.

[21] Von ENGel, A., Ionized gases ; Sd. Ed., (Clarendon Press, Oxford) 1965, p. 242-243 et 292.

[22] Druyvestein, M. J. et Penning, F. M., Rev. Mod. Phys. 12 (1940) 87 ; Howe, R. M., J. Appl. Phys. 24 (1953) 881.

[23] Phelps, A. V. et Voshall, R. E., J. Chem. Phys. 49 (1968) 3246 ; Warman, J. M., Fessenden. R. W. et Bakale, G., J. Chem. Phys. 57 (1972) 2702 ; PARKes, D. A., J. Chem. Soc., Faraday Trans. I. 68 (1972) 2103 et 2121 ; MarX, R., Mauclaire, G., Fehsenfeld, F. C., DunKIN, D. B. et Fergusson, E. E., J. Chem. Phys. 58 (1973) 3267.

[24] Fishburne, E. S. et Edse, R., J. Chem. Phys. 41 (1964) 1297 ; Gilbert, R. G. et Ross, I. G., J. Chem. Phys. 57 (1972) 2299. 